\title{
BENEFITS AND RISKS OF ICTS AND THEIR APPLICATIONS: AN ATTEMPT TO ANALYZE FREE DESCRIPTIVE ANSWERS
}

\author{
Yukiko Maruyama \\ Tokai University \\ Hiratsuka Kanagawa, Japan
}

\begin{abstract}
This paper presents the results of an attempt to analyze the benefits and risks of information and communication technologies (ICTs) and their applications, as perceived by university students. A survey was conducted using questionnaire with a free descriptive answering format. Responses were analyzed via text mining and correspondence analysis; in this manner, explanations of students' perceptions could be deduced. As the survey conducted here was preliminary, the sample size was small; further analysis with a larger number of participants is necessary.
\end{abstract}

\section{KEYWORDS}

ICT, Risk, Benefit, Text Mining, Correspondence Analysis

\section{INTRODUCTION}

Over the past several decades, various ICTs and their applications have been developed. As Brey (2018) states, "Technology has become integral to the fabric of society, and helps to shape its quality" (p.39), technology has huge impact on our society. In particular, ICTs and their applications rapidly infiltrate our society and influence our lives in various situations. It can be seen that the diffusion of ICTs simultaneously provides both advantages and disadvantages to different groups of people, possibly creating division and conflict. For this reason, we should accept ICTs carefully. While creating rules and regulations comprise one method of mediating such conflicts, legislations tend to be delayed. As such, individuals need to acquire the appropriate skills to make decisions on such issues by themselves and to participate in legislation in an informed manner. These decisions should be made not only for individual benefit but also for the benefit of society as a whole. Hence, a fundamental approach to handling this situation is to evaluate the value of every type of ICT and its applications.

Benefit and/or risk perceptions are regarded as factors that influence the acceptance of emerging technologies, even those unrelated to ICT. There have been many studies examining this (Bearth and Siegrist 2016, Liu et al. 2019, Wilson et al. 2017). While the ability to accurately perceive the benefit and risk factors of technologies is essential to evaluate their value, it is difficult to sufficiently analyze these factors. It is supposed that perceptions depend on subjective experiences and attitudes toward technologies. Sato and Tabata (2018) investigated risk perceptions to Internet use for older adults and university students; the results of their semi-structured interviews revealed notable differences in risk and benefit perceptions between the two groups. The results showed that perceived benefits and risks differed based on respondents' personal Internet usage patterns. Moreover, it is probable that the characteristics of the technologies themselves influence users' perceptions. It is possible that some users could accurately perceive the risks of certain technologies but not others.

To support people's acquisition of such situation-specific skills, education is expected to play a significant role. It is desirable to establish appropriate curriculum and lesson plans to foster students' abilities. As the first step to achieving this, it is important to understand students' current perceptions of the benefits and risks of various ICTs. To compare the students' perceived benefits and risks of multiple ICTs will show that what kind of characteristics are hardly noticed by students. As mentioned above, some 
research has investigated users' perceived benefits and risks for specific ICTs; however, there are few studies that have simultaneously investigated them for multiple ICTs. Moreover, it is difficult to gain a clear understanding of students' perceptions of benefit and risk factors for multiple ICTs. This study aims to gain a general overview of the benefits and risks of multiple ICTs and their applications as perceived by university students. Text mining for free descriptive answer text by university students and the correspondence analysis were attempted. This paper present results from the preliminary survey analysis attempt.

\section{SURVEY}

The survey used in this study was administered via a questionnaire to thirteen university students in April 2019. The participants were from an expanded minor ICT-related educational program, indicating that they had greater interest in ICT than average university students. The questionnaire included 19 questions related to the usage, risks, and benefits of ICTs (robots, self-driving cars, drones, AI, big data, and AI speakers) and 3 questions related to their consent to participating in the survey. In this paper, 12 questions related to benefit and risk of ICTs are focused on. The questions were in the following format: "What factors concerning the usage of robot (self-driving car, drone, AI, big data or AI speaker) do you think are beneficial/risky?" Participants were asked to answer by freely describing all the ideas they could think of.

Responses were input as electronic data for analysis. At the input stage, obvious typological errors were corrected. For response data related to both benefits and risks, word extraction and correspondence analysis were performed using KH Coder (Higuchi 2016, Higuchi 2017).

\section{RESULTS AND DISCUSSION}

Figure 1 shows correspondence analysis results for perceived benefits. It can be seen that "drone" and "robot" are located close to one another; and words such as "danger," "job," "work," and "use" were located close to these two items. This suggests that participants consider the availability of drones and robots for use in dangerous situations to be beneficial. In addition, "reduce," "labor cost," "people," and so on are located near "robot," and "easy," "sky," "place," "congestion," and so on are located near "drone." This allows us to conclude that participants seemed to think that these two items, especially robots, can serve as an alternative to people.

"AI" was located close to "drone" and "robot" on the Dimension 1-2 plane, but it was relatively far from these two items on the Dimension 1-3 plane. However, "big data" was located relatively close to "AI," as compared to other ICTs. "Accurate" was located close to these two items, implying that participants saw accuracy as a common benefit of AI and big data.

"AI speaker" and "self-driving car" are located far from every other item. "AI speaker" was characterized by "communication," "voice," "search," and "ask." Although an AI speaker is application of AI, participants seemed to perceive more practical benefits in it. "Self-driving car" was characterized by "reduce," "decrease," "accident," "risk," "load," "drive," "old," and "human error." Participants seemed to consider load reduction to be a benefit of self-driving cars. Unlike robots and drones, self-driving cars seem to be considered as assistants, rather than alternatives, to people.

Figure 2 shows correspondence analysis results for perceived risks. "Robot" and "self-driving car" are located closely; words such as "bug," "malfunction," "dementia," and "prevent" were located near these two items, implying that participants were concerned about technical imperfection. Moreover, they seemed to consider cognitive decline to be a risk of using robots or self-driving cars as alternatives or assistants to people.

"Drone" and "AI speaker" are located relatively close to one another. "Mischief" and "wiretap" were relatively close to these two items, implying that participants were concerned about malicious acts by users. Unlike for robots and self-driving cars, participants were more conscious of intentional threats by users.

"Big data" was characterized by "data," "personal information," and "management." Participants were more concerned with managing data than how big data is used. "AI" was located relatively far from any other words. 


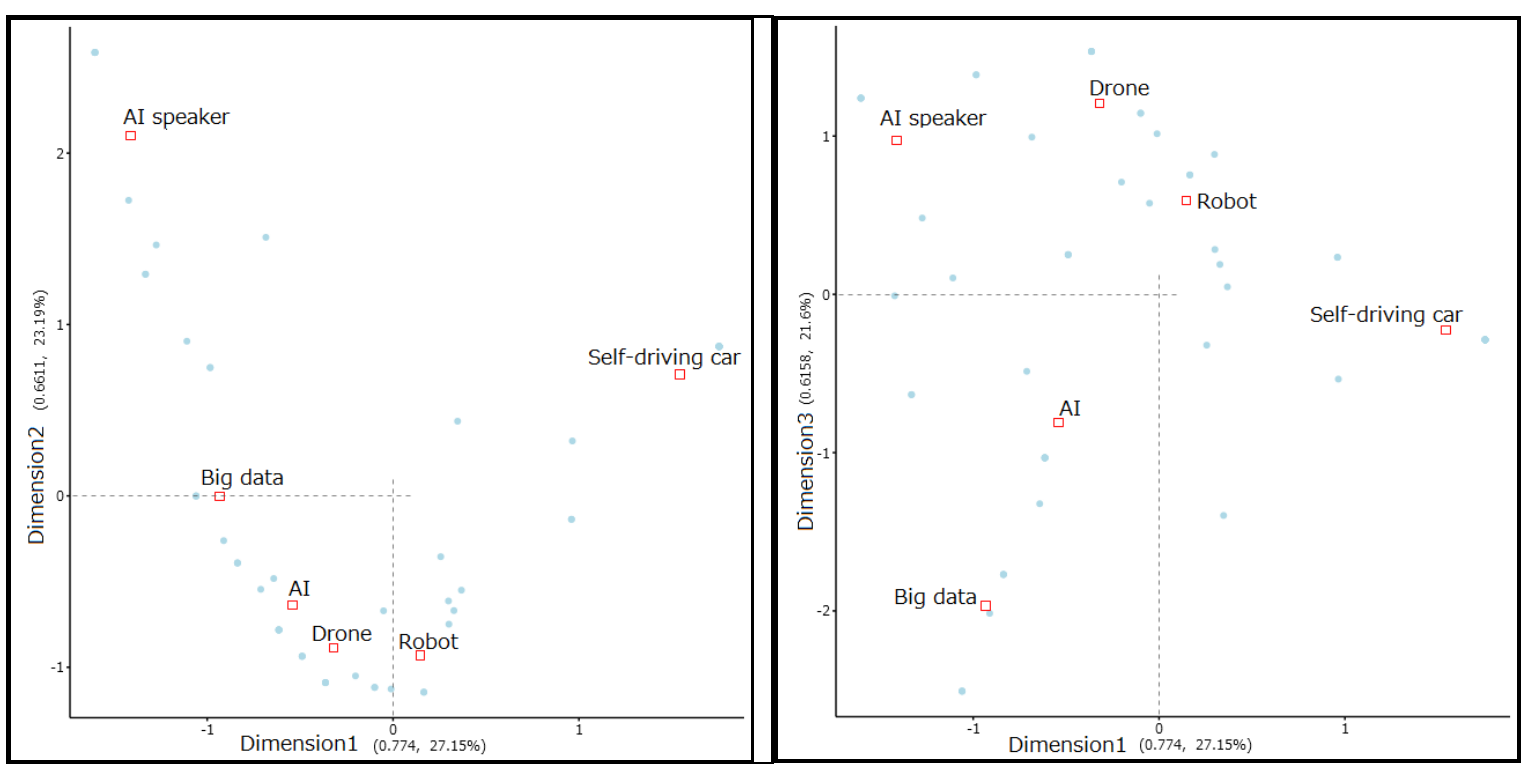

Figure 1. Results of correspondence analysis of perceived benefits for ICTs

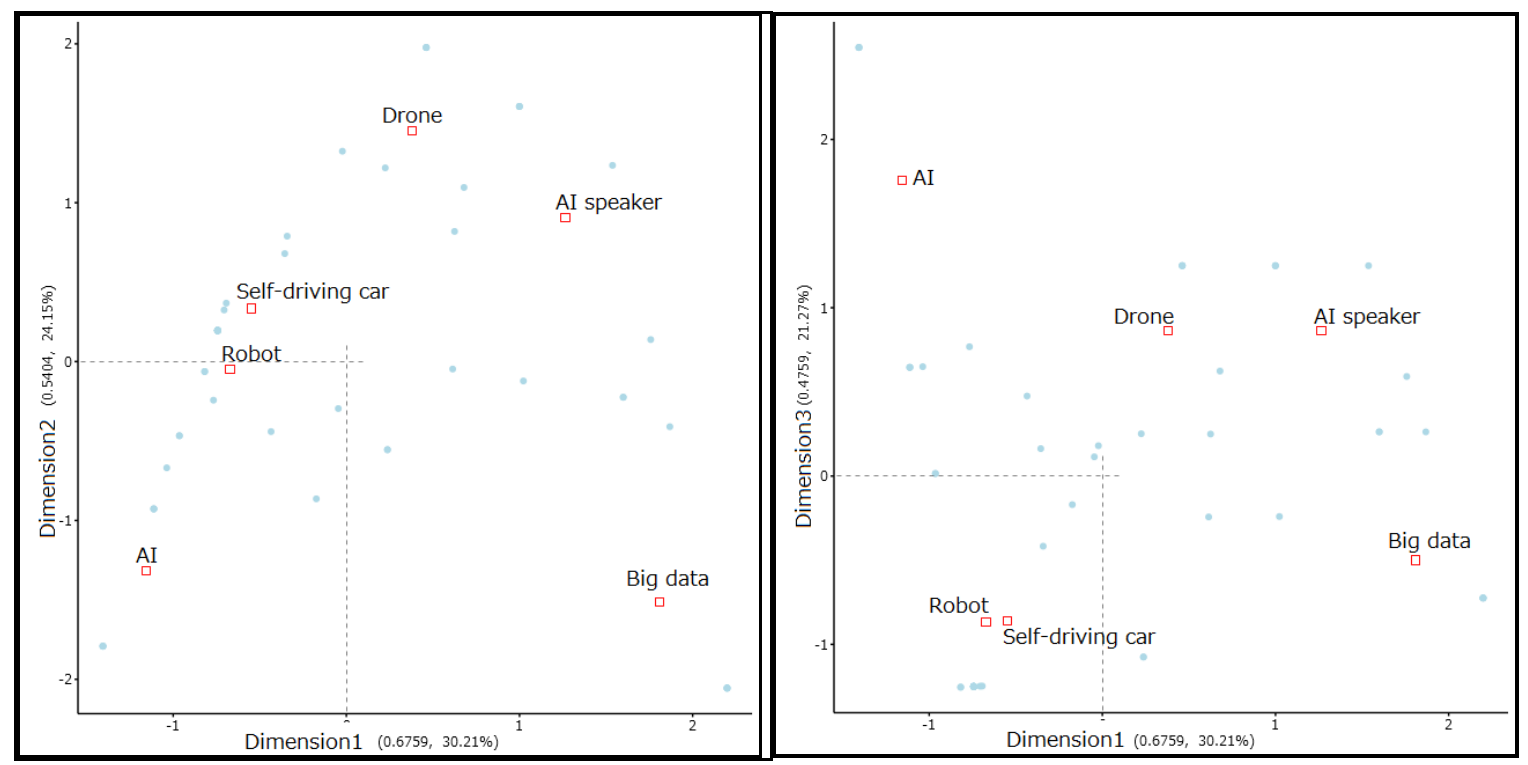

Figure 2. Results of correspondence analysis of perceived risks for ICTs

\section{CONCLUSION}

This study aims to gain a general overview of the benefits and risks perceived by university students' for multiple ICTs and their applications. Analysis of survey results was attempted using text mining and correspondence analysis. The following conclusions could be deduced from the results of our analysis.

1) Participants considered the availability for use in dangerous situations to be a benefit of drones and robots.

2) Participants saw accuracy as a common benefit of both AI and big data.

3) Participants seemed to perceive more practical benefits.

4) Participants seemed to consider the reduction of load on people to be a benefit of self-driving cars.

5) Participants were concerned about technical imperfections. 
6) Participants were more conscious of intentional threats by users in drones and AI speakers.

6) Participants were more concerned with managing data themselves than how big data is used.

The sample size in this study was small because the survey presented here was preliminary. Further analysis with a larger number of participants is necessary.

\section{REFERENCES}

Bearth, A., and Siegrist, M., 2016. A risk or benefit perceptions more important for public acceptance of innovative food technologies: A meta-analysis. Trends in Food Science \& Technology, Vol. 49, 14-23.

Brey, P., 2018. The strategic role of technology in a good society. Technology in Society, Vol. 52, pp 39-45.

Higuchi, K., 2016. A Two-Step Approach to Quantitative Content Analysis: KH Coder Tutorial Using Anne of Green Gables (Part I). Ritsumeikan Social Science Review, Vol. 52, No. 3, pp. 77-91.

Higuchi, K., 2017. A Two-Step Approach to Quantitative Content Analysis: KH Coder Tutorial Using Anne of Green Gables (Part II). Ritsumeikan Social Science Review, Vol. 53, No. 1, pp. 137-147.

Liu, P., Ma, Y. and Zuo, Y., 2019. Self-driving vehicles: Are people willing to trade risks for environmental benefits? Transportation Research Part A, Vol. 125, pp. 139-149.

Sato, H., and Tabata, N., 2018. Characteristics of risk perception to Internet use for older adults. Shinshu studies in humanities, Vol. $5 \mathrm{~m}$ pp. 29-39. (In Japanese)

Wilson, C., Hargreaves, T., and Hauxwell-Baldwin, R., 2017. Benefits and risks of smart home technologies. Energy Policy, Vol. 103, pp. 72-83. 